\title{
Raynaud's Phenomenon after COVID-19 Vaccination: Causative Association, Temporal Connection, or Mere Bystander?
}

\author{
Nikolaus Urban ${ }^{a}$ Benedikt Weber ${ }^{a}$ Julia Deinsberger ${ }^{a}$ \\ Michael Gschwandtner ${ }^{b}$ Wolfgang Bauer ${ }^{a} \quad$ Alessandra Handisurya ${ }^{a}$ \\ ${ }^{a}$ Department of Dermatology, Medical University of Vienna, Vienna, Austria; ${ }^{\circ}$ Division \\ of Angiology, Department of Internal Medicine II, Medical University of Vienna, Vienna, \\ Austria
}

\section{Keywords}

Raynaud's phenomenon - Raynaud's syndrome $\cdot$ COVID-19 vaccine $\cdot$ COVID-19 vaccination

\begin{abstract}
Herein, we report a case of a new-onset Raynaud's phenomenon (RP), which occurred in an otherwise healthy 31-year-old Caucasian woman, who lacked any known risk factors and associations with possible causes for secondary RP. However, 2 weeks prior to the development of RP, the patient had received her first injection of the COVID-19 vaccine containing ChAdOx1SARS-COV-2. The patient presented with well-demarcated, white-pale, cold areas involving the middle fingers of both hands and the ring finger of the right hand, which were triggered by exposure to cold environment and accompanied by a sensation of numbness. Infrared thermography revealed notable temperature differences of up to $10.9^{\circ} \mathrm{C}$ between affected and nonaffected fingers. Coagulation and immunological parameters, including cryoglobulins and pathological autoantibodies, were within the normal range and antibodies to the heparin/platelet factor 4 complex not detectable. It remains unclear if the development of RP in our patient is causally related to antecedent COVID-19 vaccination; however, the temporal connection to the vaccination, the complete absence of RP in her past medical history, and the lack of any risk factors and triggers raise the suspicion of a yet unknown association with the vaccine. Whether a clear association between the development of RP and COVID-19 vaccination exists or whether RP represents a bystander effect needs to be awaited in case observational reports on RP accumulate. Given the steadily rising numbers of people receiving COVID-19 vaccinations, physicians may remain alert to still unrecognized side effects.
\end{abstract}




\section{Introduction}

Raynaud's phenomenon (RP) is an episodic, vasospastic disorder affecting the small muscular arteries and arterioles of the acra, such as fingers, toes, and, less frequently, nose and ears [1]. It is characterized by a triphasic attack with color changes, starting with pallor of the skin due to vasospasm, followed by cyanosis as a result of deoxygenation and ending with rubor caused by tissue reperfusion [1]. Common trigger factors are cold temperature and emotional stress. RP affects approximately $5 \%$ of the general population, particularly women [1, 2]. Risk factors include smoking, migraine, and family history of RP [3]. RP can be classified into primary ("idiopathic") and secondary RP $[1,2]$. Primary RP is more common (80-90\%) with onset of disease usually occurring before the age of 30. Secondary RP manifests at higher ages and is associated with medical disorders, such as autoimmune rheumatic diseases, hematologic abnormalities, vascular disorders, and hypothyroidism, with certain medications, such as beta-blockers, and with prolonged and repetitive, particularly vibratory, trauma [1, 2]. Herein, we report a case of RP occurring 2 weeks after the first injection of a COVID-19 vaccine.

\section{Case Presentation}

A 31-year-old Caucasian woman presented with well-demarcated, white-pale, cold areas involving the volar and dorsal aspects of the middle fingers of both hands and the ring finger of the right hand (Fig. 1). Digital pitting, ulceration, or gangrene were not observed. The changes were transient, triggered by exposure to cold environment and accompanied by a sensation of numbness, all consistent with RP. The remaining digits lacked any abnormalities. The peripheral pulses of both arms were strong and symmetrical. The patient was otherwise healthy, did not suffer from allergies or other comorbidities, particularly autoimmune diseases, did not smoke, was not on oral contraception or other medications, and could not recall any vibratory triggers. Neither the patient nor her family had a history of RP. Two weeks prior to onset, the patient had received the first injection of the COVID-19 vaccine containing ChAdOx1-SARS-COV-2 ("Vaxzevria"; AstraZeneca AB, Sweden).

Routine blood cell counts and biochemistry as well as coagulation and immunological parameters, including antibodies to the heparin/platelet factor 4 (PF4) complex, antinuclear and extractable nuclear antibodies, complements, and cryoglobulins, were within normal ranges (Table 1). A SARS-CoV-2 antibody level of $73.8 \mathrm{U} / \mathrm{mL}$ (reference range $<0.8 \mathrm{U} / \mathrm{mL}$ ) was detected. Nailfold capillaroscopy of the affected fingers (Fig. 2A) revealed normal morphology, density, and dimensions of the capillaries, albeit minor torsions and slight dilatations were observed. Giant capillaries and hemorrhages were absent as well as signs of microangiopathy, although the blood flow was marginally reduced in the affected fingers. Infrared thermography of both hands (Fig. 2B) revealed notable temperature differences of up to $10.9^{\circ} \mathrm{C}$ between the individual fingers with $21.4^{\circ} \mathrm{C}$ and $21.2^{\circ} \mathrm{C}$ in the left index and middle finger, respectively, indicating reduced perfusion, and $32.1^{\circ} \mathrm{C}$ and $30^{\circ} \mathrm{C}$ in the right middle and ring finger, indicative of reactive hyperemia. The other digits remained at temperature levels between $26^{\circ} \mathrm{C}$ and $29^{\circ} \mathrm{C}$. In order to detect the presence of autoreactive antibodies present in the vascular tissues, particularly to the endothelium, indirect immunofluorescence employing the patient's serum was performed on normal skin and, subsequently, on cultured human endothelial cells, but yielded negative results (not shown).

The patient was advised to avoid cold exposure and other known triggers of RP and to pay attention to signs for the development of systemic sclerosis. At subsequent follow-up

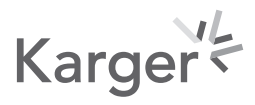




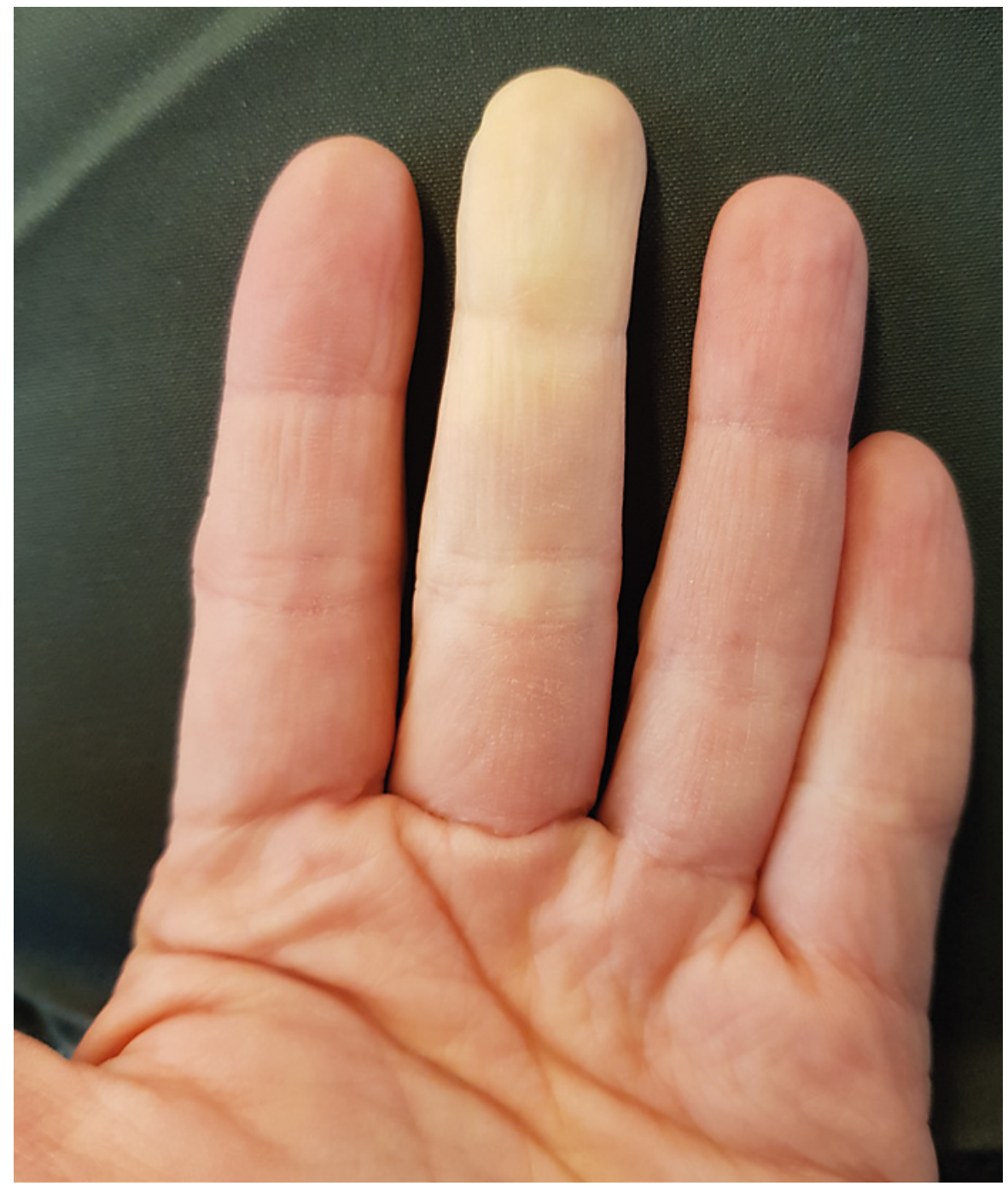

Fig. 1. Raynaud's phenomenon. Well-demarcated, white-pale, cold areas affecting the middle finger of the left hand of a 31-year old, otherwise healthy, woman.

visits after 4, 8, and 12 weeks, the patient was symptom free and did not report additional episodes. Whether this could be considered as complete recovery or attributed to the rising temperatures in spring is yet unknown. Further evaluations in the colder months are required.

\section{Discussion}

$\mathrm{RP}$ in association with vaccination is generally a rare observation. Few cases were reported on the occurrence of RP after administration of diphtheria-tetanus, hepatitis B, and human papillomavirus vaccines [4-6]. In addition, several cohort studies assessed the association between hepatitis B or human papillomavirus vaccination and the development of possible adverse events, such as RP. However, a clear association between the vaccines and the manifestation of RP could not be confirmed in cohort studies and a meta-analysis [7-10].

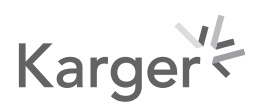


Table 1. Blood parameters of the patient with Raynaud's phenomenon after COVID-19 vaccination

\begin{tabular}{|c|c|c|}
\hline Parameter, units & $\begin{array}{l}\text { Patient's } \\
\text { value }\end{array}$ & $\begin{array}{l}\text { Reference } \\
\text { range }\end{array}$ \\
\hline RBC, T/L & 4.7 & $3.8-5.2$ \\
\hline WBC, g/L & 6.96 & $4.0-10.0$ \\
\hline Platelet count, g/L & 171 & $150-350$ \\
\hline Creatinine, mg/dL & 0.70 & $0.50-0.90$ \\
\hline AST, U/L & 13 & $<35$ \\
\hline ALT, U/L & 12 & $<35$ \\
\hline GGT, U/L & 14 & $<40$ \\
\hline Alkaline phosphatase, $\mathrm{U} / \mathrm{L}$ & 55 & $35-105$ \\
\hline LDH, U/L & 151 & $<250$ \\
\hline $\mathrm{CRP}, \mathrm{mg} / \mathrm{dL}$ & $<0.03$ & $<0.5$ \\
\hline D-dimer, $\mu \mathrm{g} / \mathrm{mL}$ & 0.30 & $<0.5$ \\
\hline Fibrinogen, mg/dL & 237 & $200-400$ \\
\hline Prothrombin time, $\%$ & 109 & $75-130$ \\
\hline aPTT, s & 28.9 & $27.0-41.0$ \\
\hline TT, s & 15.6 & $<21$ \\
\hline Plasminogen activity, \% & 104 & $65-200$ \\
\hline $\begin{array}{l}\text { Heparin/platelet factor } 4 \text { complex (IgG) antibodies } \\
\text { (optical density) }\end{array}$ & $\begin{array}{l}\text { Negative } \\
(0.02)\end{array}$ & $\begin{array}{l}\text { Negative } \\
(<0.51)\end{array}$ \\
\hline Cryofibrinogen & Negative & Negative \\
\hline Cryoglobulin & Negative & Negative \\
\hline ANA & Negative & $<1: 80$ \\
\hline Anti-dsDNA antibodies, IU/mL & $<0.6$ & $<10$ \\
\hline $\begin{array}{l}\text { ENA subsets (SSA [Ro], SSB [La], SCL-70, SM, u1RNP, Jo-1, } \\
\text { centromer-B, cardiolipin IgG, cardiolipin IgM, } \\
\text { beta-2-glycoprotein IgG, beta-2-glycoprotein IgM) }\end{array}$ & Negative & Negative \\
\hline IgG, mg/dL & 874 & $700-1600$ \\
\hline IgA, mg/dL & 92 & $70-400$ \\
\hline IgM, mg/dL & 131 & $40-230$ \\
\hline $\mathrm{C} 3 \mathrm{c}, \mathrm{mg} / \mathrm{dL}$ & 92 & $90-180$ \\
\hline $\mathrm{C} 4, \mathrm{mg} / \mathrm{dL}$ & 16.4 & $10-40$ \\
\hline $\mathrm{CH} 50, \mathrm{U} / \mathrm{mL}$ & $>60$ & $31.6-57.6$ \\
\hline Total protein, g/L & 69.6 & $64-83$ \\
\hline Albumin, g/L & 49.4 & $35-52$ \\
\hline Alpha-1 globulin, \% & 4.0 & $2.9-4.9$ \\
\hline Alpha-2 globulin, \% & 8.7 & $7.1-11.8$ \\
\hline Beta-1 globulin, \% & 5.7 & $4.7-7.2$ \\
\hline Beta-2 globulin, \% & 3.8 & $3.2-6.5$ \\
\hline Gamma globulin, \% & 12.5 & $11.1-18.8$ \\
\hline Paraprotein & Negative & Negative \\
\hline $\mathrm{TSH}, \mu \mathrm{IU} / \mathrm{mL}$ & 1.60 & $0.27-4.2$ \\
\hline
\end{tabular}


Table 1 (continued)

\begin{tabular}{lll}
\hline Parameter, units & $\begin{array}{l}\text { Patient's } \\
\text { value }\end{array}$ & $\begin{array}{l}\text { Reference } \\
\text { range }\end{array}$ \\
\hline Free T3, pg/mL & 3.28 & $2.15-4.12$ \\
Free T4, ng/dL & 1.21 & $0.76-1.66$ \\
\hline
\end{tabular}

RBC, red blood cells; WBC, white blood cells; AST, aspartate aminotransferase; ALT, alanine aminotransferase; GGT, gamma-glutamyl transpeptidase; LDH, lactic acid dehydrogenase; CRP, C-reactive protein; aPTT, activated partial thromboplastin time; TT, thrombin time; ANA, antinuclear antibodies; TSH, thyroid-stimulating hormone; free $\mathrm{t} 3$, free triiodothyronine; free $\mathrm{T} 4$, free thyroxine.

The COVID-19 pandemic has swept across the world, and since then, global efforts were made to rapidly develop protective vaccines. While the available COVID-19 vaccines are protective against symptomatic COVID-19-infections, the entire spectrum of possible adverse effects is not yet known due to the accelerated approval of the vaccines and the limited observational period. In the past months, reports of thrombosis, primarily cerebral and splanchnic venous thrombosis, in association with thrombocytopenia and coagulation disorders occurring few weeks after ChAdOx1-SARS-COV-2 vaccination have raised much interest and led to revision of the list of side effects of this vaccine [11].

The underlying mechanisms causative for RP in our patient are not known, but could be mediated by changes in coagulation and blood viscosity, triggering of an (auto) immunological cascade, neural factors, or a not yet elucidated pathway. In our patient, thrombocyte counts, coagulation parameters, D-dimer, and fibrinogen levels were within physiological range. No evidence for the presence of cryoglobulins or pathological autoantibodies was found, suggesting that these immunological mechanisms were not responsible for the onset of RP. Furthermore, anti-PF4/heparin antibodies, which are known to induce Fc-receptormediated platelet activation and enhance thrombin generation resulting in thrombocytopenia and thrombosis $[12,13]$, were not detected. However, evaluation was performed about 7 weeks after vaccination. Although anti-PF4 antibodies are of an IgG type, the levels tend to wane rapidly, becoming undetectable after a median of 50-85 days [14]. While we cannot exclude the possibility that low levels of anti-PF4/heparin antibodies were elicited after vaccination and have vanished within the first 6-7 weeks, this scenario seems unlikely.

Considering the high prevalence of primary RP among women in this age group, the development of RP in our patient could also be unrelated to prior COVID-19 vaccination. However, the complete absence of RP in the past medical history, the lack of any risk factors and associations with possible causes for secondary RP, and the temporal connection to COVID-19 vaccination raise the suspicion of a yet unknown association with the vaccine. Given the steadily rising numbers of people receiving COVID-19 vaccinations, physicians may remain alert to still unrecognized side effects. Whether a clear association between development of RP and COVID-19 vaccination exists or whether RP represents a bystander event needs to be awaited in case observational reports on RP accumulate.

\section{Statement of Ethics}

The authors have no ethical conflicts to disclose. The patient gave written informed consent for publication of the case and accompanying images. The study was conducted in accordance with the World Medical Association Declaration of Helsinki. The case report was exempt from approval of the Ethics Committee of the Medical University of Vienna.

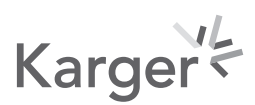



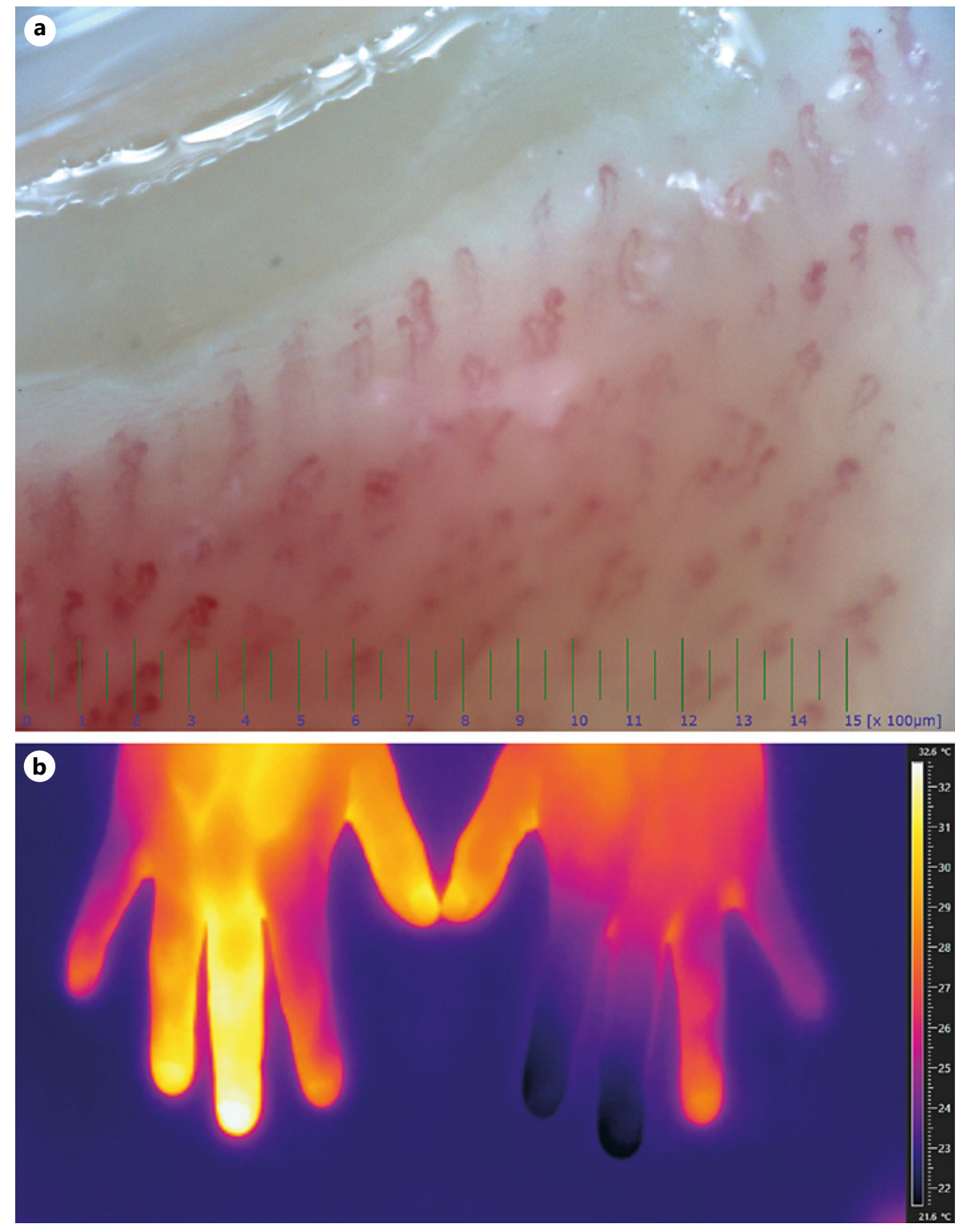

Fig. 2. A Nailfold capillaroscopy of the middle finger of the left hand showing normal morphology, density, and dimensions of the capillaries. B Thermogram of both hands, revealing temperature differences of up to $10.9^{\circ} \mathrm{C}$ between the individual fingers with $21.4^{\circ} \mathrm{C}$ and $21.2^{\circ} \mathrm{C}$ in the left index and middle finger, respectively, indicating reduced perfusion, and $32.1^{\circ} \mathrm{C}$ and $30^{\circ} \mathrm{C}$ in the right middle and ring finger, indicative of reactive hyperemia.

\section{Conflict of Interest Statement}

The authors have no conflicts of interest to declare.

\section{Funding Sources}

Austrian Science Fund, Grant number P29619-B30. 
Urban et al.: Raynaud's Phenomenon after COVID-19 Vaccination

\section{Author Contributions}

Drs. Nikolaus Urban, Benedikt Weber, Julia Deinsberger, and Alessandra Handisurya contributed to conception, design, interpretation, and manuscript preparation. Drs. Michael Gschwandtner and Wolfgang Bauer contributed to acquisition, analysis, and interpretation of data for the work and critically revising of the manuscript for important intellectual content. All authors read and approved the final manuscript and agreed to be accountable for all aspects of the work in ensuring that questions related to the accuracy or integrity of any part of the work are appropriately investigated and resolved.

\section{Data Availability Statement}

All data generated or analyzed during this study are included in this article. Further enquiries can be directed to the corresponding author.

\section{References}

1 Haque A, Hughes M. Raynaud's phenomenon. Clin Med. 2020 Nov;20(6):580-7.

2 Wigley FM, Flavahan NA. Raynaud's Phenomenon. N Engl J Med. 2016 Aug 11;375(6):556-65.

3 Garner R, Kumari R, Lanyon P, Doherty M, Zhang W. Prevalence, risk factors and associations of primary Raynaud's phenomenon: systematic review and meta-analysis of observational studies. BMJ Open. 2015 Mar 16; 5(3): e006389.

4 Aksay A. A Raynaud phenomonic attack induced after an adult diphtheria-tetanus vaccine. Haydarpasa Numune Med J. 2019;59(3):296-8. Duzgol.

5 Selvaraj V, Ogunneye 0, Lagu T, Ryzewicz S. A rare case of Raynaud's vasculitis secondary to hepatitis B vaccination: the induced auto-immune attack syndrome. Case Rep Int Med. 2014;1(1):17-20.

6 Tomljenovic L, Colafrancesco S, Perricone C, Shoenfeld Y. Postural orthostatic tachycardia with chronic fatigue after HPV vaccination as part of the "autoimmune/auto-inflammatory syndrome induced by adjuvants": case report and literature review. J Investig Med High Impact Case Rep. 2014 Mar 18;2(1):2324709614527812.

7 Bouquet É, Urbanski G, Lavigne C, Lainé-Cessac P. Unexpected drug-induced Raynaud phenomenon: analysis from the French national pharmacovigilance database. Therapie. 2017 Oct;72(5):547-54.

8 Prete M, Favoino E, Giacomelli R, Afeltra A, Cantatore FP, Bruno C, et al. Evaluation of the influence of social, demographic, environmental, work-related factors and/or lifestyle habits on Raynaud's phenomenon: a casecontrol study. Clin Exp Med. 2020 Feb;20(1):31-7.

9 Hviid A, Svanström H, Scheller NM, Grönlund O, Pasternak B, Arnheim-Dahlström L. Human papillomavirus vaccination of adult women and risk of autoimmune and neurological diseases. J Intern Med. 2018 Feb;283(2): 154-65.

10 Willame C, Gadroen K, Bramer W, Weibel D, Sturkenboom M. Systematic review and meta-analysis of Postlicensure Observational Studies on human papillomavirus vaccination and autoimmune and other rare adverse events. Pediatr Infect Dis J. 2020 Apr;39(4):287-93.

11 European Medicines Agency. Vaxzevria (previously COVID-19 Vaccine AstraZeneca). EPAR - Product Information; 2021. Available from: https://www.ema.europa.eu/en/documents/product-information/vaxzevriapreviously-covid-19-vaccine-astrazeneca-epar-product-information_en.pdf.

12 Scully M, Singh D, Lown R, Poles A, Solomon T, Levi M, et al. Pathologic antibodies to platelet factor 4 after ChAdOx1 nCoV-19 vaccination. N Engl J Med. 2021 Jun 10;384(23):2202-11.

13 Greinacher A, Thiele T, Warkentin TE, Weisser K, Kyrle PA, Eichinger S. Thrombotic thrombocytopenia after ChAdOx1 nCov-19 vaccination. N Engl J Med. 2021 Jun 3;384(22):2092-101.

14 Warkentin TE, Kelton JG. Temporal aspects of heparin-induced thrombocytopenia. N Engl J Med. 2001 Apr 26; 344(17):1286-92. 